\title{
The influence of learning strategies in the acquisition, retention, and transfer of a procedural task
}

\author{
ROBERT N. SINGER, GENE KORIENEK, and SUSAN RIDSDALE \\ Florida State University, Tallahassee, Florida 32306
}

\begin{abstract}
In order to analyze the effectiveness of various learner strategies upon initial learning, retention, and subsequent transfer of a motor skill, 50 college-aged subjects were randomly assigned to one of five strategy conditions: imagery, chunking, verbalization, informed choice, and control. The task, a serial manipulation apparatus (SMA), required subjects to manipulate a predetermined sequence of buttons and switches. Following learning trials, subjects were administered a retention test. Additionally, a transfer task was administered in order to discern the generalizability of specific strategies. Separate analyses for errors by positions, total errors, and total time revealed that the informed-choice group performed better on the acquisition task than did the chunking, verbalization, or imagery groups, whereas the imagery group performed better on the transfer task than did the chunking, verbalization, and informed-choice groups.
\end{abstract}

In recent years, researchers have shown an increased interest in the cognitive processes that may primarily influence the learning of various matters. This thrust, in turn, has generated numerous approaches to examine the effectiveness of a number of learner strategies to facilitate the acquisition and retention of information. In our own work (e.g., Singer \& Gerson, 1979), a strategy is interpreted as a self-initiated or externally imposed way of directing information leading to decisions for purposeful behavior.

The implication for the field of motor learning would appear to be that the use of appropriate strategies should facilitate the learning process. However, while there exists an abundance of supportive evidence for strategy usage within the verbal learning area (Bevan \& Steger, 1971; Bower \& Winzenz, 1970; Paivio \& Yuille, 1969), research is severely lacking within the motor learning domain. Thus, inferences must be drawn from the verbal learning research as to the potential beneficial effects of various types of strategies on the acquisition and retention of motor skills.

While the use of strategies has been shown to facilitate the acquisition and retention of newly learned material (e.g., Campione \& Brown, 1974; Kendler, 1964; Kendler \& Kendler, 1962), the generalizability (transfer) of these same strategies to different situational contexts is questionable. As Gagné (1977) has pointed out, the difficulty lies in arranging conditions so that transfer can be demonstrated. Strategy transfer usually cannot occur unless the initial learning environment includes some

This research was supported by the Defense Advance Research Projects Agency. Requests for reprints should be sent to Robert N. Singer, Movement Science-Physical Education Department, Florida State University, Tallahassee, Florida 32306. reference to the transfer situation (Bransford, Franks, Morris, \& Stein, 1978; Campione \& Brown, 1974), such as the temporal structuring of the components within the task being similar (Keele \& Summers, 1976). Campione and Brown (1974) suggested that transfer is best when the form of the two situations remains the same. More specifically, context may well direct one's attention toward relevant facts. A familiar problem in a new context fails to elicit any strategy, since nothing in the situation cues the learner how to approach the problem. To overcome this circumstance, Campione (1973) suggested that when individuals learn useful problem solving strategies, these strategies should be employed in a variety of situations so that a particular strategy is not restricted to a specific context. It would appear, then, that strategies that enhance skill acquisition and retention also have the potential to transfer to the learning of a skill in a new situation with similar parameters.

It was the purpose in the present study to analyze the effectiveness of specific strategies on the acquisition, retention, and subsequent transfer of a procedural task involving sequential motor responses. The following strategy conditions were investigated:imagery, chunking, rote verbalization, and informed choice. While there exists little evidence to support the effectiveness of strategy usage for a procedural task, it would appear that either imagery or chunking (Housner \& Hoffman, Note 1; Ridsdale, Note 2) would enable a learner to acquire initial skill more effectively. Therefore, it was hypothesized that during acquisition and retention trials, subjects in the imagery and chunking groups would perform better than the rote verbalization group.

Although the potential for the transfer of strategies from one task to another is evident, relatively few 
investigations have dealt specifically with this area. However, it would appear that strategies that are compatible with the learner's cognitive style (Pask, 1975; Ridsdale, Note 2) may be more amenable to transfer situations. More specifically, imposed strategies may enhance initial learning and retention; however, transfer situations require an individual to identify the existent similarities between tasks. Thus a self-imposed strategy, consistent with the learner's cognitive style, may be more easily applied in transfer tasks. Therefore, it was hypothesized that subjects in the informed-choice group would display a greater degree of transfer learning between the acquisition and transfer phase of the investigation.

\section{METHOD}

\section{Subjects}

Fifty male and female undergraduate and graduate students (mean age $=20.74$ years, $S D=3.50$ ) from Florida State University volunteered to participate in this study. The subjects were randomly assigned to five groups.

\section{Apparatus}

The Serial Manipulation Apparatus (SMA) (cf. Singer, 1976) is a computer-managed task that engages two computer systems for operation. A Control Data Cyber 74 stores the software that is used to generate the control programs for the SMA, and an IMSAI 8080 microprocessor actually controls program operation and data collection. A Sony CUM-51WWD video monitor was used to allow the experimenter to monitor the subject's progress and the operation of the apparatus.

The hardware portion of the SMA is a wood and metal structure, with a horizontal platform situated at table-top height. A rectangular encasement in the back of the SMA houses a Radio Shack TRS-80 video monitor that was used to display visual instructions, stimuli, and knowledge of results to the subjects. A Panasonic RQ-413AS audio cassette tape player/recorder was used to provide taped instructions to the subjects.

A number of response manipulanda in the form of switches and levers were situated in various locations throughout the task display. A "home" button was situated in the center of this section and nearest the front edge. In the present study, the home button had to be depressed by the subject to initiate and terminate the response sequence. Each response mechanism had a lightemitting diode (LED) placed next to it; this served as an additional visual cue to the subjects.

\section{Procedure}

Subjects entered the test area individually and were randomly assigned a strategy condition. The strategy groups were: (1) imagery, with subjects instructed to mentally picture storage bins in a warehouse and to "place" each response mechanism into a particular bin; (2) chunking, with subjects told to group the responses into sequences of three responses each; (3) rote verbalization, with subjects required to repeat each stimulus aloud as the response was made; (4) informed choice, with subjects instructed in the use of the three previous strategies and then told to select one of them, a combination of them, or a strategy of their own; and (5) a control group, in which subjects were not provided specific strategy instructions.

The subjects received two familiarization trials designed to acquaint them with the apparatus and subsequent procedure. Prior to engagement in the acquisition trials, the subjects received taped instructions on the use of the particular strategy that was randomly assigned to them. Following strategy instructions, they received two practice trials with the same responses that were to be used during the actual learning phase. On these practice trials, subjects were cued as to the correct response through the video monitor and the LED next to each switch.

After the two practice trials, subjects performed eight acquisition trials from memory. No cues were available on the screen, nor were the LED's lit. Depression of the home button at the end of each sequence caused the feedback information to appear on the screen. Feedback information consisted of the subject's reaction time, movement time, total response time, position errors, and total response errors for each trial. If the button was pushed in the middle of the sequence or a mechanism was manipulated out of order, an "error" message was flashed on the screen. The message remained there until the correct response was made. When all nine responses were made in the correct order and the home button was pressed, one trial was completed.

Subjects were required to finish eight acquisition trials, after which half the subjects in each strategy group rested during the intertrial interval and the 20 -sec retention interval; the other half performed a written addition task during the same time period. Following this interval, subjects performed the same sequence of responses with no external visual cues as a test of retention. The retention test was followed by a 2-min rest interval, at which time subjects were questioned as to their use of a particular strategy. These verbal reports were recorded on a brief, specially constructed questionnaire. Upon completion of the rest interval, subjects began the transfer phase of the task.

\section{RESULTS}

A 5 by 2 by 8 by 9 (strategies by test conditions by trials by positions) factorial ANOVA, with repeated measures on the last three factors, was conducted on response errors that occurred at each position. A significant trials effect $[F(7,315)=37.96, p<.01]$ was revealed, with fewer errors generally emitted with practice. There was also a significant positions effect $[F(8,360)=7.38, p<.01]$. Follow-up comparisons revealed that more errors were made at Position 6 than at any other position. Additionally, both Positions 4 and

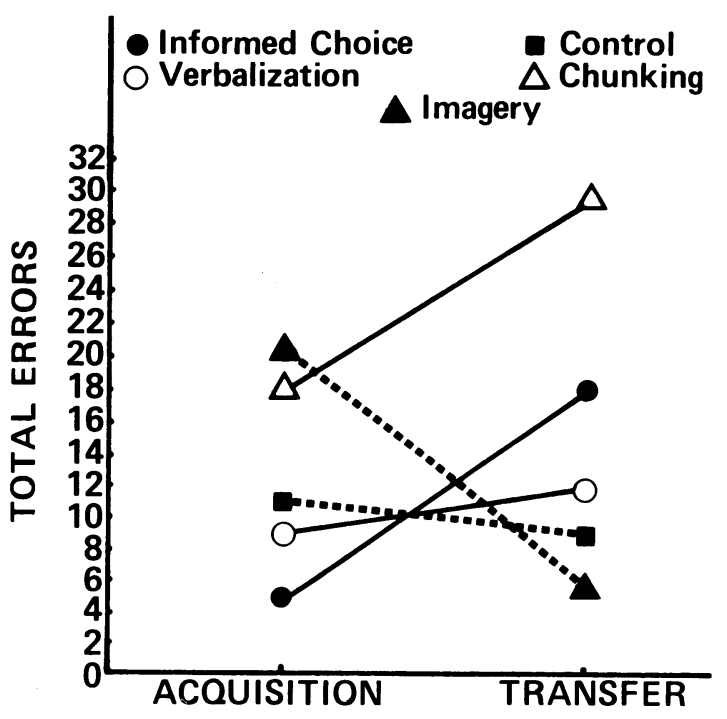

Figure 1. Total errors compared among groups between acquisition and transfer phases. 


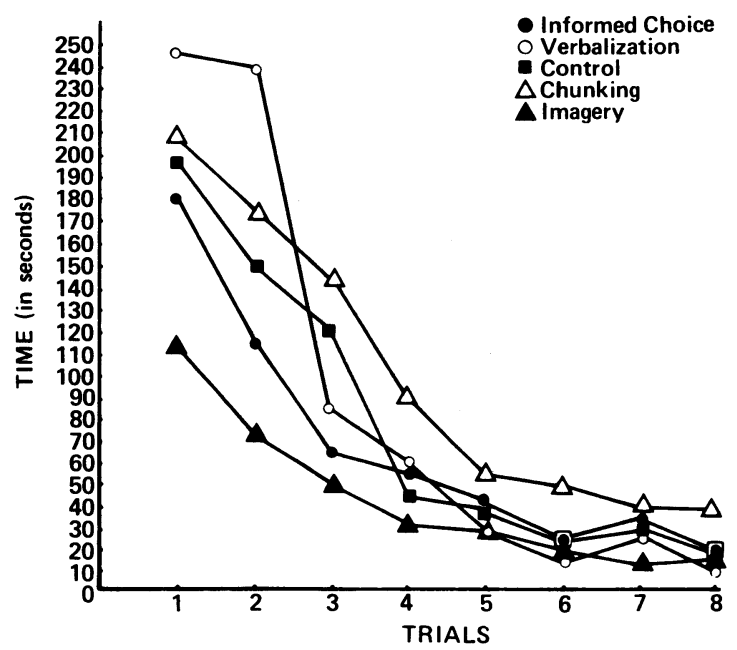

Figure 2. Task completion time for groups across trials.

5 resulted in more response errors than any other positions except 6 and 8 , respectively, indicative of a primacy-recency effect. A significant Test by Strategies interaction $[\mathrm{F}(4,45)=3.11, \mathrm{p}<.05]$ is depicted in Figure 1.

A 5 by 2 by 8 (strategies by test conditions by trials) factorial ANOVA, with repeated measures on the last two factors, was conducted on the total errors made on each trial. A significant Test Conditions by Strategy interaction $[F(4,45)=3.10, p<.05]$ was also revealed. In addition, a significant trials main effect $[F(7,315)=$ $36.97, \mathrm{p}<.01]$ was revealed, indicative of a learning effect across trials.

The final dependent variable analyzed was time to task completion. A 5 by 2 by 8 (strategies by test conditions by trials) factorial ANOVA, with repeated measures on the last two factors, yielded a significant trials effect $[F(7,315)=57.84, p<.01]$ (see Figure 2). There was also a significant strategy effect $[F(4,45)=$ $2.77, \mathrm{p}<.05]$, with the imagery group taking the least time to complete the task and the chunking group, the longest.

A 5 by 2 by 2 (strategies by retention interval by trials) ANOVA was conducted to determine retention effects, with total errors as the dependent measure. A 5 by 3 by 2 by 8 (strategies by retention interval by trials by positions) ANOVA was conducted, with number of errors made at each position as the dependent measure. And a 5 by 2 by 2 ANOVA was run, with time as the dependent variable. All failed to indicate any significant differences among the groups.

\section{DISCUSSION}

The initial hypothesis predicting a better performance by the imagery and chunking groups than by the verbalization, informed-choice, or control groups was not supported by the data collected; however, the significant reduction in both total errors and time to complete the task was clearly indicative of a general learning effect. Similarly, the primacy-recency effect manifested by a greater number of errors at the middle positions is consistent with results reported within the verbal learning literature (Murdock, 1962).

It was also predicted that the informed-choice group would display the greatest degree of transfer between the acquisition and transfer phases of the experiment. Although the data do not support this prediction, they do suggest several possible interpretations. It is known that various imposed strategies may be utilized to facilitate the rate of initial skill acquisition (Singer \& Pease, 1976); however, the same strategies may not enhance skill learning in transfer situations (Singer \& Gaines, 1975). In line with this view, it has been suggested that, in certain situations, imposed strategies may be in competition with preexistent strategies within the learner's repertoire (Pask, 1975). This notion could lend some credence to the superior performance of the control group in both the acquisition and transfer phases of the present experiment.

Since the subjects in the control group were given no strategy instructions, it may be logically assumed that they incorporated a (self-generated) strategy consistent with their own cognitive styles. In addition, questionnaire data administered after both acquisition and retention trials revealed that subjects in the control group consistently employed the same strategy across both acquisition and transfer tasks. However, subjects in the chunking, verbalization, and informed-choice groups reported that they changed strategies during both acquisition and transfer phases. The questionnaire data indicated that many of the subjects in the verbalization, informed-choice, and control groups either started with or changed to forms of imagery or chunking after the first few trials of the experiment. This tendency of the subjects to switch to forms of chunking and imagery is reflected strongly by the similar performances of all groups in Trials 4-8.

An alternative explanation for the lack of strategy transfer may be due to the similarity of the acquisition and transfer tasks. The two tasks (acquisition and transfer) were identical in every aspect, excluding the specific switch positions. It appears as though the transfer task developed by changing the positions to be learned was not sufficiently different to avoid similarity interference. The fact that the imagery group demonstrated superior performance during the transfer task suggests that they may have been incorporating additional cuing. Indeed, questionnaire results revealed that in addition to imaging location, imagery subjects also thought about the sequence between positions.

Results of the present investigation are reflective of an obvious need to emphasize the learning of a strategy before application is required (Singer, Gerson, \& Ridsdale, Note 3). In this sense, strategy instruction must be sufficient in communication to enable the learner to fully comprehend its usage and to use it continuously throughout a given task. The potential plasticity and transience of the cognitive processes activated during strategy utilization present a considerable challenge for investigators to deal with. The prerequisites of measurement assume that these processes remain fixed and unchanging during the period of measurement; however, this is obviously not the case. It is necessary to incorporate additional experimental controls in order to increase the reliability of the measurements made. This would, in effect, decrease the variability of the data collected. Perhaps when this occurs the true potential value of learning strategies as an aid in skill acquisition and transfer, as speculated elsewhere (Singer, 1978; Singer et al., Note 3), will be realized.

\section{REFERENCE NOTES}

1. Housner, L., \& Hoffman, S. J. Imagery ability in recall of distance and location information. Paper presented at the meeting of the Canadian Society for Psychomotor Learning and Sport Psychology, Toronto, Ontario, Canada, November 1978. 
2. Ridsdale, S. The enhancement of skill acquisition through learner strategies. Unpublished manuscript, Florida State University, 1978.

3. Singer, R. N., Gerson, R. F., \& Ridsdale, S. The effect of various strategies on the acquisition, retention, and transfer of a serial positioning task (Tech. Rep. 399). Washington, D.C: U.S. Army Research Institute for the Behavioral and Social Sciences, July 1979.

\section{REFERENCES}

Atrinson, R. C., Hansen, D. N., \& Bernbach, H. A. Short term memory with young children. Psychonomic Science, 1964, $1,225-256$.

Bevan, W., \& Steger, J. A. Free recall and abstractness of stimuli. Science, 1971, 172, 597-599.

Bransford, J. D., Franks, J. J., Morris, C. D., \& Stein, B. S. Some general constraints on learning and memory research. In. F. I. M. Craik \& L. S. Cermak (Eds.), Levels of processing and theories of memory. Hillsdale, N.J: Erlbaum, 1978.

Bower, G. H., \& Winzenz, D. Comparison of associative learning strategies. Psychonomic Science, 1970, 20, 119-120.

Campione, H. C. The generality of transfer: Effects of age and similarity of training and transfer tasks. Journal of Experimental Child Psychology, 1973, 15, 407-418.

Campione, J. C., \& Brown, A. L. The effects of contextual changes and degree of component mastery on transfer training. In $\mathrm{H}$. W. Reese (Ed.), Advances in child development and behavior. New York: Academic Press, 1974.

GAGNE, R. N. Conditions of learning. N.Y: Holt, Rinehart, \& Winston, 1977.

KeEle, S. W., \& Summers, J. J. The structure of motor programs. In G. E. Stelmach (Ed.), Motor control: Issues and trends.
N.Y: Academic Press, 1976.

Kender, T. S. Verbalization and optional reversal shifts among kindergarten children. Journal of Verbal Learning and Verbal Behavior, 1964, 3, 428-436.

Kendler, H. H., \& Kendler, T. S. Vertical and horizontal processes in problem solving. Psychological Review, 1962, 69, 1-16.

MuRdock, B. B. The serial position effect of free recall. Journal of Experimental Psychology, 1962, 64, 482-488.

Paivio, A. M., \& Yuille, J. C. Changes in associative strategies and paired-associate learning over trials as a function of word imagery and type of learning set. Journal of Experimental Psychology, 1969, 79, 458-463.

PASK, G. The cybernetics of human learning and performance. London: Hutchinson, 1975.

SingER, R. N. The serial manipulation apparatus. Journal of Motor Behavior, 1976, 8, 69-73.

Singer R. N. Motor skills and learning strategies. In H. F. O'Neil, Jr. (Ed.), Learning strategies I. New York: Academic Press, 1978.

Singer, R. N., \& Gaines, L. Effect of prompted and trialand-error learning on transfer performance of a serial motor task. American Educational Research Journal, 1975, 12, 395-403.

Singer, R. N., \& Gerson, R. F. Learning strategies, cognitive processes, and motor learning: In. H. F. O'Neil \& C. D. Spielberger (Eds.), Cognitive and affective learning strategies. New York: Academic Press, 1979.

Singer, R. N. \& Pease, D. The effect of different instructional strategies on learning, retention, and transfer of a serial motor task. Research Quarterly, 1976, 47, 788-796.

(Received for publication June 12, 1980.) 